\title{
Robust profit opportunities in risky financial portfolios
}

\author{
Mustafa Ç. Pınar ${ }^{\mathrm{a}}$, Reha H. Tütüncǘ ${ }^{\mathrm{b}, *, 1}$ \\ ${ }^{a}$ Department of Industrial Engineering, Bilkent University, 06800 Ankara, Turkey \\ ${ }^{\mathrm{b}}$ Department of Mathematical Sciences, Carnegie Mellon University, Pittsburgh, PA 15213, USA
}

Received 6 January 2004; accepted 12 August 2004

Available online 26 October 2004

\begin{abstract}
For risky financial securities with given expected return vector and covariance matrix, we propose the concept of a robust profit opportunity in single- and multiple-period settings. We show that the problem of finding the "most robust" profit opportunity can be solved as a convex quadratic programming problem, and investigate its relation to the Sharpe ratio.

(C) 2004 Elsevier B.V. All rights reserved.
\end{abstract}

Keywords: Financial securities; Arbitrage; Robust optimization; Sharpe ratio

\section{Introduction and background}

Existence and exclusion issue of arbitrage in financial markets is a well-studied area of mathematical finance treated at different levels of detail in several research monographs and textbooks; see e.g., [5,10,13].

The purpose of the present paper is (1) to introduce a novel concept related to arbitrage which we call a robust profit opportunity for risky financial contracts (or, securities for short) when the investor has access to the expected return and standard deviation data (or, perhaps an estimate thereof) of the securities, (2) to develop simple optimization models that compute the

\footnotetext{
* Corresponding author.

E-mail address: reha@math.cmu.edu (R.H. Tütüncü).

${ }^{1}$ Work was supported by National Science Foundation under Grants CCR-9875559 and DMS-0139911.
}

most robust profit opportunities in single-period and multi-period investment environments, and, (3) to relate these ideas to the maximum Sharpe ratio problem.

The main finance contribution of the paper is to propose a new investment concept strongly related to arbitrage using partial probabilistic information, and to show that the proposed model is computationally tractable as it involves the solution of convex quadratic programs that are routinely and efficiently solved by polynomial-time interior point methods. In this sense, although we introduce a more general model than the classical discrete arbitrage model, computationally, the new model is, in theory and practice, no more difficult than the classical theory which involves the use of linear programming duality.

Consider a single-period decision environment with a set of $n$ risky financial securities. Let $v_{i}$ denote the period-end value of $\$ 1$ invested in security $i$ at the 
beginning of the period. Let $v=\left(v_{1}, \ldots, v_{n}\right) \in \mathbf{R}^{n}$ denote the vector of end-of-period values. Treating $v$ as a random vector, let us denote its expected value by $\bar{v}$ and its $n \times n$ (symmetric, positive semidefinite) matrix of variance/covariances by $Q$. We assume that $\bar{v}$ is not a positive multiple of $e$, the $n$-dimensional vector of ones to avoid degenerate cases. We define $r=v-e$ to be the vector of returns and $\bar{r}=\bar{v}-e$ denotes its expected value. Next, we let $x \in \mathbf{R}^{n}$ represent a portfolio of the $n$ securities where $x_{i}$ corresponds to the amount (in dollars) invested in security $i$. Then, for a given $x$ the total investment in this portfolio will be $e^{\mathrm{T}} x=\sum_{i} x_{i}$ and the value of the portfolio at the end of the period is a random variable, namely, $v^{\mathrm{T}} x$.

Let $\tilde{v}$ be a particular realization of the random variable $v$ revealed to the investor at the end of the period. If the investor knew $\tilde{v}$ at the beginning of the period, she could make money if there exists a portfolio $x$ such that $\tilde{v}^{\mathrm{T}} x \geqslant 0, e^{\mathrm{T}} x<0$. In other words, if there is a portfolio that can be formed with a negative investment and that achieves a non-negative value at the end of the period, the investor can make money. Of course, since the first inequality depends on random quantities, such a portfolio does not represent an arbitrage opportunity.

In contrast, a portfolio $x$ that satisfies

$\bar{v}^{\mathrm{T}} x \geqslant 0, \quad x^{\mathrm{T}} Q x=0, \quad e^{\mathrm{T}} x<0$,

corresponds to an arbitrage opportunity since the condition $\operatorname{Var}(x)=x^{\mathrm{T}} Q x=0$ indicates that the final portfolio value is actually non-random and equal to its non-negative expected value. If we assume that arbitrage opportunities do not exist, we conclude that the system (1) must be inconsistent. Now, let us act as a conservative investor who recognizes that a sure profit as in (1) is not possible but is seeking a highly likely profit opportunity at the end of a single investment period. Further assume that the investor believes that a random number is "rarely" less than its mean minus a positive scalar $\theta$ times its standard deviation. In the absence of arbitrage, such an investor may be satisfied if the following condition is satisfied.

There exists a portfolio $x$ such that

$\bar{v}^{\mathrm{T}} x-\theta \sqrt{x^{\mathrm{T}} Q x} \geqslant 0, \quad e^{\mathrm{T}} x<0$.

The quantity $\bar{v}^{\mathrm{T}} x-\theta \sqrt{x^{\mathrm{T}} Q x}$ is related to the notion of risk-adjusted return for the portfolio $x$ where $\theta$ cor- responds to a measure of risk-aversion of the investor. It is also reminiscent of the 2-sigma or 3-sigma engineering approach - these would correspond to choices of $\theta=2$ or $\theta=3$. As we argue below, system (2) is related to the robust optimization approach of BenTal and Nemirovski [3,2], and with this motivation we call portfolios satisfying (2) robust profit opportunities with $\theta$ representing the level of robustness, and we call the problem (3) below the maximum- $\theta$ robust profit opportunity problem.

We note that a weaker version of (2) is obtained by relaxing the strict inequality:

There exists a portfolio $x$ such that $\bar{v}^{\mathrm{T}} x-$ $\theta \sqrt{x^{\mathrm{T}} Q x} \geqslant 0, \quad e^{\mathrm{T}} x \leqslant 0$.

This relaxation is meaningful only with additional constraints since $x=0$ is a feasible vector for these inequalities for all values of $\theta$.

To motivate the development of system (2) as in [3] let us assume that the future values $v_{1}, v_{2}, \ldots, v_{n}$ fall within the uncertainty intervals $\Delta_{i}=\left[\bar{v}_{i}-\sigma_{i}, \bar{v}_{i}+\sigma_{i}\right]$. Assume, furthermore, that $v_{i}$ 's are mutually independent and symmetrically distributed in $\Delta_{i}$ with respect to the mean value $\bar{v}_{i}$. For a fixed choice of portfolio holdings $x$, the end-of-period portfolio value can be expressed as $P=\sum_{i=1}^{n} \bar{v}_{i} x_{i}+\varsigma$, where $\varsigma=\sum_{i=1}^{n} x_{i}\left(v_{i}-\bar{v}_{i}\right)$ has zero mean and variance $\operatorname{Var}(\varsigma)=\sum_{i=1}^{n} x_{i}^{2} \mathrm{E}\left\{\left(v_{i}-\bar{v}_{i}\right)^{2}\right\}$. Since the variance of $v_{i}$ is bounded above by $\sigma_{i}^{2}$ one has $\operatorname{Var}(\varsigma) \leqslant V(x) \equiv$ $\sum_{i=1}^{n} x_{i}^{2} \sigma_{i}^{2}$.Therefore, one can say that typically the value of $P$ will differ from the mean value of $\bar{v}^{\mathrm{T}} x$ by a quantity proportional to $\sqrt{\operatorname{Var}(\varsigma)} \leqslant \sqrt{V(x)}$, variations on both sides being equally probable. Therefore, choosing a reliability coefficient $\theta$ and ignoring all events where the random future value is less than $\bar{v}^{\mathrm{T}} x-\theta \sqrt{V(x)}$, one arrives at the robust profit opportunity definitions introduced above. Notice that by ignoring the events where the future portfolio value is less than $\bar{v}^{\mathrm{T}} x-\theta \sqrt{V(x)}$, one accepts the fact that $\operatorname{Prob}(\varsigma<-\theta \sqrt{V(x)})<\mathrm{e}^{-\theta^{2} / 2}$ as shown in [4]. The right-hand side is getting already quite small (in the order of $10^{-7}$ for $\theta=6$ ) quickly with increasing values of $\theta$. Therefore, the larger the scalar $\theta$, the smaller the risk. Therefore, in Section 2 we will be looking for portfolios $x$ that satisfy (2) for the largest possible $\theta$ :

$\sup _{\theta, x} \theta, \quad$ s.t. $\bar{v}^{\mathrm{T}} x-\theta \sqrt{x^{\mathrm{T}} Q x} \geqslant 0, \quad e^{\mathrm{T}} x<0$. 
Notice that, in addition to being nonlinear and not differentiable everywhere, the first constraint in (3) is non-convex in $\theta$ and $x$ when $\theta$ is a variable and therefore (3) is a non-convex optimization problem. Consequently, at first glance it appears that our model is intractable. Exploiting the homogeneity of the constraints, we show below that this problem can in fact be reduced to a convex quadratic programming problem and obtain a closed form solution. We also derive extensions of our results to multi-period settings.

The rest of this paper is organized as follows. In the next few paragraphs, we present some connections of our robust profit opportunity (RPO) model to existing literature. In Section 2, we formulate the maximum- $\theta$ RPO problem, establish a convex quadratic programming equivalent of this problem and demonstrate its solution. In Section 3, we relate the maximum- $\theta$ RPO problem to the maximum Sharpe ratio problem. In Section 4 we develop a two-period RPO model without a riskless asset. Finally, a two-period model including a riskless asset is studied in Section 5.

\subsection{Connections to previous work}

The current paper is built on an earlier work of the first author [12]. While this earlier paper focused on the feasibility problem (2) for a fixed $\theta$ and analyzed the existence of its solutions using conic duality, our focus here is on the optimization problem (3) and its reduction to a convex quadratic programming problem.

An interesting connection exists between the concepts we introduced above and the following wellknown concepts, the value-at-risk formula [6,7], chance constrained optimization [14], and robust optimization paradigm of Ben-Tal and Nemirovski $[3,2]$. In fact, the present paper is motivated by the contributions of Ben-Tal and Nemirovski. Let us begin by briefly reviewing the robust optimization approach. Our treatment in this section closely follows Section 2.6 of [11].

We want to find a vector $x \in \mathbf{R}^{n}$ that satisfies $v^{\mathrm{T}} x \geqslant 0$. This, of course, is an easy task for any given $v \in \mathbf{R}^{n}$. We consider a decision environment where $v$ is not known exactly, but is known to belong to an uncertainty set $\mathscr{E}$. In this case, a "robust" version of the inequality $v^{\mathrm{T}} x \geqslant 0$ is the following system:

$v^{\mathrm{T}} x \geqslant 0$, for all $v \in \mathscr{E}$.

When $\mathscr{E}$ is an ellipsoidal uncertainty set, e.g., $\mathscr{E}=\{\bar{v}+$ $\left.\bar{L} u:\|u\|_{2} \leqslant 1\right\}$ with $\bar{v} \in \mathbf{R}^{n}$ and $\bar{L}$ an $n \times k$ matrix, we have that (4) is equivalent to $\min _{v \in \mathscr{E}} v^{\mathrm{T}} x \geqslant 0=$ $\min _{u:\|u\|_{2} \leqslant 1} \bar{v}^{\mathrm{T}} x+u^{\mathrm{T}} \bar{L}^{\mathrm{T}} x \geqslant 0$. It is easy to see that the optimal $u$ is given by $u^{*}=-\bar{L}^{\mathrm{T}} x /\left\|\bar{L}^{\mathrm{T}} x\right\|$. Letting $Q=\left(1 / \theta^{2}\right) \bar{L} \bar{L}^{\mathrm{T}}$, we see that the above inequality is identical to the first inequality in (2).

Alternatively, we can consider the assumption that the uncertain vector $v$ is actually a Gaussian random vector, with mean $\bar{v}$ and covariance $Q$. We may require as in [14] that the inequality $v^{\mathrm{T}} x \geqslant 0$ should hold with a confidence level exceeding $\eta$, for some given $\eta \geqslant 0.5$, i.e., $\operatorname{Prob}\left(v^{\mathrm{T}} x \geqslant 0\right) \geqslant \eta$. Defining $u=v^{\mathrm{T}} x, \bar{u}=\bar{v}^{\mathrm{T}} x$, and $\sigma=\sqrt{x^{\mathrm{T}} Q x}$ one can normalize both sides of the inequality as follows:

$\operatorname{Prob}\left(\frac{u-\bar{u}}{\sigma} \geqslant \frac{-\bar{u}}{\sigma}\right) \geqslant \eta$.

Since $(u-\bar{u}) / \sigma$ is a zero mean, unit variance Gaussian random variable the above probability constraint is simply equivalent to $-\bar{u} / \sigma \leqslant \Phi^{-1}(1-\eta)=-\Phi^{-1}(\eta)$, where $\Phi(z)=(1 / \sqrt{2 \pi}) \int_{-\infty}^{z} \mathrm{e}^{-t^{2} / 2} \mathrm{~d} t$ is the CDF of a zero mean, unit variance Gaussian random variable. Now, constraint (5) is nothing other than $\bar{v}^{\mathrm{T}} x-$ $\Phi^{-1}(\eta) \sqrt{x^{\mathrm{T}} Q x} \geqslant 0$. Since we assumed that $\eta \geqslant 0.5$, $\Phi^{-1}(\eta)$ is a non-negative scalar. The close resemblance to the first inequality of (2) is now obvious. The above tail probability concepts are also reminiscent of the value-at-risk methodology used to limit the risk exposure of financial institutions [6]. A recent study on portfolio optimization with the worst-case valueat-risk criterion using conic programming is [7].

We can go one step further and ask that the inequality $v^{\mathrm{T}} x \geqslant 0$ should hold with the largest possible confidence level $\eta$, i.e., ask that the lower bound on $\operatorname{Prob}\left(v^{\mathrm{T}} x \geqslant 0\right)$ is maximized. Since the function $\Phi^{-1}(\eta)$ is monotone increasing between 0 and 1 , we obtain a problem analogous to (3).

We note that there has been an intensive study of robust optimization formulations for asset allocation problems in recent years, see, e.g., [11,3,7,8]. While our approach shares the intuitive notion of robustness with the models in these papers and is related to value-at-risk and Sharpe ratio maximization (see 
Section 3), our model differs significantly from these approaches. Unlike the robust optimization models mentioned above, we do not consider the expected return and covariance information to be uncertain. We take these values as given and certain and seek portfolios that provide a next best alternative to arbitrage opportunities. Our contributions lie in the conversion of the resulting seemingly intractable problems into convex quadratic programs whose analytic solutions can be readily derived and, perhaps more importantly, in the extension of these results to multi-period settings.

\section{Minimum risk robust profit opportunities}

Recall the maximum- $\theta$ RPO problem we formulated in the previous section:

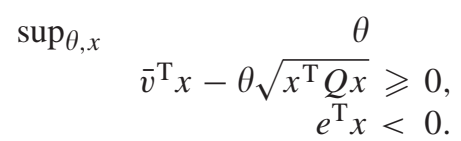

Now, we will transform this non-convex optimization problem into a convex quadratic programming problem. For the remainder of this section, we assume that the matrix $Q$ is nonsingular, and hence is positive definite. This is essentially equivalent to assuming that there are no redundant assets (those that can be perfectly replicated by the remaining assets) or risk-free assets in the collection of securities we consider.

Since $Q$ is positive definite, $x^{\mathrm{T}} Q x>0$ for all nonzero $x$ and therefore, $\bar{v}^{\mathrm{T}} x-\theta \sqrt{x^{\mathrm{T}} Q x} \geqslant 0$ if and only if $\bar{v}^{\mathrm{T}} x / \sqrt{x^{\mathrm{T}} Q x} \geqslant \theta$ for all nonzero $x$. Therefore, problem (6) is equivalent to the following problem:

$$
\begin{aligned}
& \sup _{x} \frac{\bar{v}^{\mathrm{T}} x}{\sqrt{x^{\mathrm{T}} Q x}} \\
& e^{\mathrm{T}} x<0 .
\end{aligned}
$$

This is an optimization problem with a nonlinear, and possibly non-concave, objective function. We note that if $x$ is feasible for (7), then so is $\kappa x$ for any $\kappa>0$, and the objective function value is constant along such feasible rays. Since the objective function and the constraint are homogeneous in $x$ introducing a constraint that normalizes the $x$ variables will not affect the optimal value as long as the hyperplane defined by this constraint intersects the cone of optimal solutions. This is similar to the technique used by Goldfarb and
Iyengar in solving the robust maximum Sharpe ratio problem [8].

Let us introduce the normalizing constraint $\bar{v}^{\mathrm{T}} x=1$. Since we assumed that $\bar{v}$ is not a positive multiple of $e$, there exists vectors $x$ such that $\bar{v}^{\mathrm{T}} x>0$ and $e^{\mathrm{T}} x<0$ and we can conclude that the optimal objective value of (7) is positive. There are three possibilities: (i) the optimal value is positive, bounded, and is achieved on the feasible set, (ii) the optimal value is positive and bounded but is achieved only on the boundary of the (open) feasible set, (iii) the objective function is unbounded above. In all three cases, adding the constraint $\bar{v}^{\mathrm{T}} x=1$ does not alter the behavior of the solutions, i.e., either there exists an optimal $x^{*}$ such that $\bar{v}^{\mathrm{T}} x^{*}=1$ in the feasible set or its closure, or there exists a sequence of points $x^{k}$ such that $\bar{v}^{\mathrm{T}} x^{k}=1$ and the objective function grows indefinitely as $k \rightarrow \infty$. Consequently, problem (7) is equivalent to

$$
\begin{aligned}
& \sup _{x} \frac{\bar{v}^{\mathrm{T}} x}{\sqrt{x^{\mathrm{T}} Q x}} \\
& e^{\mathrm{T}} x<0, \\
& \bar{v}^{\mathrm{T}} x=1
\end{aligned}
$$

or

$$
\begin{aligned}
& \sup _{x} \frac{1}{\sqrt{x^{\mathrm{T}} Q x}}, \\
& e^{\mathrm{T}} x<0, \\
& \bar{v}^{\mathrm{T}} x=1
\end{aligned}
$$

or

$$
\begin{aligned}
& \inf _{x} \frac{1}{2} x^{\mathrm{T}} Q x, \\
& e^{\mathrm{T}} x<0, \\
& \bar{v}^{\mathrm{T}} x=1,
\end{aligned}
$$

where we introduced the factor $\frac{1}{2}$ for convenience. We formally state this equivalence in the next proposition.

Proposition 1. The maximum- $\theta$ RPO problem (6) is equivalent to the convex quadratic optimization problem (8). When optimal solutions exist, for any optimal solution $\left(x^{*}, \theta^{*}\right)$ of (6) we have that $\left[1 /\left(\bar{v}^{\mathrm{T}} x^{*}\right)\right] x^{*}$ is optimal for (8), and for any optimal solution $x^{*}$ of (8) and for any $\kappa>0$ we have that $\left(\kappa x^{*}, \bar{v}^{\mathrm{T}} x^{*} / \sqrt{\left(x^{*}\right)^{\mathrm{T}} Q x^{*}}\right)$ is optimal for (6). 
Relaxing the strict inequality $e^{\mathrm{T}} x<0$ in (8) to $e^{\mathrm{T}} x \leqslant 0$, we obtain a standard convex quadratic programming problem:

$\min _{x} \frac{1}{2} x^{\mathrm{T}} Q x$

$e^{\mathrm{T}} x \leqslant 0$,

$\bar{v}^{\mathrm{T}} x=1$.

Note that we are able to replace inf with min, since the continuous objective function which tends to $\infty$ as $\|x\| \rightarrow \infty$ will necessarily achieve its minimal value over the closed feasible set. The optimality conditions of this problem are given next: $x^{*}$ is an optimal solution for the relaxed problem (9) if and only if there exists scalars $\lambda \geqslant 0$ and $\gamma$ such that

$Q x^{*}+\lambda e-\gamma \bar{v}=0$,

$e^{\mathrm{T}} x^{*} \leqslant 0$,

$\lambda\left(e^{\mathrm{T}} x^{*}\right)=0$,

$\bar{v}^{\mathrm{T}} x^{*}=1$.

Since $Q$ is positive definite, the objective function is strictly convex and the optimal solution is unique. Since, we converted the strict inequality $e^{T} x<0$ in (8) to a loose inequality, we are interested in characterizing the cases where the optimal solution to (9) actually satisfy this inequality strictly. We have the following simple result.

Proposition 2. The unique optimal solution $x^{*}$ to (9) satisfies $e^{\mathrm{T}} x^{*}<0$ if and only if $e^{\mathrm{T}} Q^{-1} \bar{v}<0$.

Proof. If $e^{\mathrm{T}} Q^{-1} \bar{v}<0$, we easily see that optimality conditions (10) are satisfied when $x^{*}=$ $\left[1 /\left(\bar{v}^{\mathrm{T}} Q^{-1} \bar{v}\right)\right] Q^{-1} \bar{v}, \lambda=0$, and $\gamma=1 /\left(\bar{v}^{\mathrm{T}} Q^{-1} \bar{v}\right)$. Therefore, $x^{*}$ is the unique optimal solution and $e^{\mathrm{T}} x^{*}=\left(e^{\mathrm{T}} Q^{-1} \bar{v}\right) /\left(\bar{v}^{\mathrm{T}} Q^{-1} \bar{v}\right)<0$.

Conversely, if $e^{\mathrm{T}} x^{*}<0$, from the complementarity equation in (10) we see that $\lambda$ must equal zero. Therefore, from the first equation we obtain $x^{*}=\gamma Q^{-1} \bar{v}$, and substituting this into the last equation in (10), we obtain $\gamma=1 /\left(\bar{v}^{\mathrm{T}} Q^{-1} \bar{v}\right)$. Then, $e^{\mathrm{T}} x^{*}=\left(e^{\mathrm{T}} Q^{-1} \bar{v}\right) /\left(\bar{v}^{\mathrm{T}} Q^{-1} \bar{v}\right)<0$ implies that $e^{\mathrm{T}} Q^{-1} \bar{v}<0$.

In the alternative case, i.e., when $e^{\mathrm{T}} Q^{-1} \bar{v} \geqslant 0$, we must have $e^{\mathrm{T}} x^{*}=0$. Using this equation, we solve the optimality system (10) and obtain:

$x^{*}=\gamma Q^{-1} \bar{v}-\lambda Q^{-1} e$,

$\gamma=\frac{e^{\mathrm{T}} Q^{-1} e}{\Delta}$,

$\lambda=\frac{e^{\mathrm{T}} Q^{-1} \bar{v}}{\Delta}$,

where

$$
\Delta=\left(e^{\mathrm{T}} Q^{-1} e\right)\left(\bar{v}^{\mathrm{T}} Q^{-1} \bar{v}\right)-\left(e^{\mathrm{T}} Q^{-1} \bar{v}\right)^{2}>0 .
$$

The positivity of $\Delta$ follows from the Cauchy-Schwartz inequality and the assumption that $e$ and $\bar{v}$ are not collinear. Since $\Delta$ is positive, both $\gamma$ and $\lambda$ are nonnegative.

In the case when $e^{\mathrm{T}} Q^{-1} \bar{v} \geqslant 0$, the optimal value of (6) is

$$
\frac{\bar{v}^{\mathrm{T}} x^{*}}{\left(x^{*}\right)^{\mathrm{T}} Q x^{*}}=\frac{1}{\sqrt{\gamma}}=\sqrt{\bar{v}^{\mathrm{T}} Q^{-1} \bar{v}-\frac{\left(e^{\mathrm{T}} Q^{-1} \bar{v}\right)^{2}}{e^{\mathrm{T}} Q^{-1} e}} .
$$

While this optimal value cannot be achieved in (6) we can get a feasible solution to (6) whose objective value is arbitrarily close to the expression in (15). Similar statements hold for problem (8); its optimal value, which is the same as that of (9) is not achieved but we can get arbitrarily close to it. In fact, consider a vector $\delta$ that satisfies $e^{\mathrm{T}} \delta<0$ and $\bar{v}^{\mathrm{T}} \delta=0$. Then, the vector $x(\varepsilon)=x^{*}+\varepsilon \delta$ is feasible for (8) for all $\varepsilon>0$ and its objective value is $\Theta(\varepsilon)=\varepsilon \delta^{\mathrm{T}} Q x^{*}+\frac{1}{2} \varepsilon^{2} \delta^{\mathrm{T}} Q \delta$ away from the optimal objective value obtained in (9).

We summarize our results in this section in the following proposition.

Proposition 3. Consider the maximum- $\theta$ RPO problem given in (6). Assuming that $Q$ is positive definite and $\bar{v}$ is not a multiple of e, the optimal value of this problem is given as follows:

$$
\theta^{*}= \begin{cases}\sqrt{\bar{v}^{\mathrm{T}} Q^{-1} \bar{v}} & \text { if } e^{\mathrm{T}} Q^{-1} \bar{v}<0, \\ \sqrt{\bar{v}^{\mathrm{T}} Q^{-1} \bar{v}-\frac{\left(e^{\mathrm{T}} Q^{-1} \bar{v}\right)^{2}}{e^{\mathrm{T}} Q^{-1} e}} & \text { if } e^{\mathrm{T}} Q^{-1} \bar{v} \geqslant 0 .\end{cases}
$$

In the first case, this optimal value is achieved for any positive multiple of $x^{*}=Q^{-1} \bar{v}$. In the second case, the optimal value is not achieved but feasible perturbations of $x^{*}$ given in (11) come arbitrarily close to this value. 


\section{Relation to the Sharpe ratio}

In this section we treat the case where there is a riskless security with return $r_{f}>0$ available for investment in addition to the $n$ risky securities we considered above. Let $v_{f}=1+r_{f}$ denote the end-of-period value of $\$ 1$ invested in the riskless security at the beginning of the period. Since we are considering a riskless security, the full correlation matrix is no longer positive definite but we still assume that the submatrix corresponding to the risky securities is positive definite.

Let us assume that the current price of the riskless security is $\$ 1$. Then, zero-investment portfolios can be constructed by purchasing the portfolio $x$ after borrowing $e^{\mathrm{T}} x$ dollars at the riskless rate $r_{f}$ (or lending $-e^{\mathrm{T}} x$ dollars if $\left.e^{\mathrm{T}} x<0\right)$. We can represent such zeroinvestment portfolios as $\left(x,-e^{\mathrm{T}} x\right)$. Recalling that $\bar{r}=$ $\bar{v}-e$ denotes the expected return vector for the risky securities, we observe that the expected return of this zero-investment portfolio is $\bar{r}^{\mathrm{T}} x-r_{f}\left(e^{\mathrm{T}} x\right)$. A classical problem in finance is to find the zero-investment portfolio with the highest expected return to standard deviation ratio (a scale invariant quantity) - the so-called Sharpe ratio:

$\max _{x} \frac{\bar{r}^{\mathrm{T}} x-r_{f}\left(e^{\mathrm{T}} x\right)}{\sqrt{x^{\mathrm{T}} Q x}}$.

Let us call (16) the maximum Sharpe ratio problem. Since the objective function of this problem is scale invariant, the canonical representation of the problem uses the normalizing constraint $e^{\mathrm{T}} x=1$ and has

$$
\max _{x} \frac{\bar{r}^{\mathrm{T}} x-r_{f}}{\sqrt{x^{\mathrm{T}} Q x}}=\frac{\left(\bar{r}-r_{f} e\right)^{\mathrm{T}} x}{\sqrt{x^{\mathrm{T}} Q x}}
$$

$e^{\mathrm{T}} x=1$.

Equivalently, this second representation can be obtained by letting $x_{i}$ denote the "proportion of the portfolio invested in security $i$ " rather than "dollars invested in security $i$ ". The vector $\left(\bar{r}-r_{f} e\right)$ represents the "risk premium" vector for the risky securities.

Now we relate the maximum- $\theta$ (RPO) problem to the maximum Sharpe ratio problem. Consider the maximum- $\theta$ RPO problem in this case. We have the variable vector $\tilde{x}=\left(x, x_{f}\right)$, with expected value vector $\tilde{v}=\left[\bar{v} ; v_{f}\right]$ and covariance matrix

$\tilde{Q}=\left[\begin{array}{ll}Q & 0 \\ 0 & 0\end{array}\right]$

The maximum- $\theta$ RPO problem is

$$
\begin{aligned}
& \sup _{x} \frac{\tilde{v}^{\mathrm{T}} \tilde{x}}{\sqrt{\tilde{x}^{\mathrm{T}} \tilde{Q} \tilde{x}}}=\frac{\bar{v}^{\mathrm{T}} x+v_{f} x_{f}}{\sqrt{x^{\mathrm{T}} Q x}}, \\
& e^{\mathrm{T}} \tilde{x}=e^{\mathrm{T}} x+x_{f}<0,
\end{aligned}
$$

which, after relaxing the strict inequality, can be rewritten as

$$
\begin{aligned}
& \max _{x} \frac{\bar{v}^{\mathrm{T}} x+v_{f} x_{f}}{\sqrt{x^{\mathrm{T}} Q x}}, \\
& e^{\mathrm{T}} x+x_{f} \leqslant 0 .
\end{aligned}
$$

Since $Q$ is positive definite, we do not need to worry about division by zero in (17). The problematic case of $x^{\mathrm{T}} Q x=0$ occurs only when $x=0$-all feasible solutions with $x=0$ have non-positive objective values and cannot be optimum and therefore can be ignored. Also note that for a fixed $x$ the objective function is maximized by maximizing $x_{f}$. Therefore, for an optimal solution vector $\tilde{x}=\left(x, x_{f}\right)$ the constraint $e^{\mathrm{T}} x+x_{f} \leqslant 0$ will always be tight and we can replace this inequality with an equality. Now, substituting $x_{f}=-e^{\mathrm{T}} x$, we obtain $\bar{v}^{\mathrm{T}} x+v_{f} x_{f}=\bar{v}^{\mathrm{T}} x-v_{f}\left(e^{\mathrm{T}} x\right)=(\bar{r}+e)^{\mathrm{T}} x-(1+$ $\left.r_{f}\right)\left(e^{\mathrm{T}} x\right)=\bar{r}^{\mathrm{T}} x-r_{f}\left(e^{\mathrm{T}} x\right)$. Thus, (17) is equivalent to $\max _{x} \frac{\bar{r}^{\mathrm{T}} x-r_{f}\left(e^{\mathrm{T}} x\right)}{\sqrt{x^{\mathrm{T}} Q x}}$,

which is identical to (16). In other words, when the universe of investment options includes a risk-free security, portfolios that are maximum- $\theta$ RPOs coincide with maximum Sharpe ratio portfolios. With this interpretation, we also conclude that when there are no risk-free investment options, our characterization of "minimum risk" robust profit opportunities represent a generalization of the maximum Sharpe ratio portfolios.

\section{A two-period model}

Our discussion on RPOs in the preceding sections focused on single-period models. Here we extend the 
notion of RPOs to a two-period investment model. For ease of exposition further extension of the ideas below in a setting with more than two periods is not included here.

We consider the following setting. The investor will form a portfolio at time 0 that she will hold until time 1 at which point she will be able to rebalance her portfolio in a self-financing manner possibly incurring transaction costs and hold this new portfolio until time 2. We use the following notation: Let $v_{i}^{1}$ denote the (random) time 1 value of $\$ 1$ invested in security $i$ at time 0 . Similarly, let $v_{i}^{2}$ denote the (random) time 2 value of $\$ 1$ invested in security $i$ at time 1 . Let $x_{i}^{0}$ and $x_{i}^{1}$ denote the dollars invested in security $i$ at times 0 and 1 , respectively. Let $v^{1}=\left[v_{1}^{1}, \ldots, v_{n}^{1}\right]^{\mathrm{T}}$, define $v^{2}, x^{0}, x^{1}$ similarly. Then, the initial (time 0 ) value of the portfolio formed at time 0 is $e^{\mathrm{T}} x^{0}$. This portfolio has value $\left(v^{1}\right)^{\mathrm{T}} x^{0}$ at time 1 , before it is rebalanced. In the absence of transaction costs, the self-financing constraint can be posed as

$e^{\mathrm{T}} x^{1}=\left(v^{1}\right)^{\mathrm{T}} x^{0}$.

Let $\bar{v}^{2}$ and $Q_{2}$ denote the expected value vector and the covariance matrix for the random vector $v^{2}$. Then, a two-period analog of the maximum- $\theta$ robust profit opportunity problem can be posed as follows:

$$
\begin{aligned}
& \sup _{\theta, x^{0}, x^{1}} \theta, \text { s.t. } e^{\mathrm{T}} x^{0}<0, \quad e^{\mathrm{T}} x^{1}=\left(v^{1}\right)^{\mathrm{T}} x^{0}, \\
& \left(\bar{v}^{2}\right)^{\mathrm{T}} x^{1}-\theta \sqrt{\left(x^{1}\right)^{\mathrm{T}} Q_{2} x^{1}} \geqslant 0 .
\end{aligned}
$$

Unlike (6) in Section 2, this problem is not a deterministic optimization problem because of the random $v^{1}$ term in the equality constraint. However, at the time we need to choose $x^{1}$, we will have already observed this random quantity and therefore, the decision problem at time 1 is a deterministic problem. This twostep decision process with a random constraint was addressed in the adjustable robust optimization (ARO) models of Ben-Tal et al. [1,9]. These models intend to choose the decision variables in such a way that the performance of the system under the worst-case realization of the uncertain input parameters is optimized. They are called "adjustable" since some of the variables can be chosen after the uncertain parameters are observed.
Let $\mathscr{U}$ denote the set of all possible realizations of the random vector $v^{1}$. Then, the ARO model for problem (18) can be written as follows:

$$
\begin{aligned}
& \sup _{x^{0}: e^{\mathrm{T}} x^{0}<0} \inf _{v^{1} \in \mathscr{U}} \sup _{\theta, x^{1}} \theta \text { s.t. } e^{\mathrm{T}} x^{1}=\left(v^{1}\right)^{\mathrm{T}} x^{0}, \\
& \left(\bar{v}^{2}\right)^{\mathrm{T}} x^{1}-\theta \sqrt{\left(x^{1}\right)^{\mathrm{T}} Q_{2} x^{1}} \geqslant 0 .
\end{aligned}
$$

To be able to solve this problem, let us first focus on the inner maximization problem. Given $\alpha=\left(v^{1}\right)^{\mathrm{T}} x^{0}$, we want to solve

$\sup _{\theta, x^{1}} \theta$ s.t. $e^{\mathrm{T}} x^{1}=\alpha, \quad\left(\bar{v}^{2}\right)^{\mathrm{T}} x^{1}-\theta \sqrt{\left(x^{1}\right)^{\mathrm{T}} Q_{2} x^{1}} \geqslant 0$.

Let us assume as before that $Q_{2}$ is positive definite. This assumption precludes the availability of a riskfree security and will be removed in the next section. Given this assumption, we can rewrite the above problem as

$$
P(\alpha) \quad \sup _{x^{1}} \frac{\frac{\left(\bar{v}^{2}\right)^{\mathrm{T}} x^{1}}{\sqrt{\left(x^{1}\right)^{\mathrm{T}} Q_{2} x^{1}}}}{e^{\mathrm{T}} x^{1}}=\alpha .
$$

Unlike (6), the constraint of (20) is not homogeneous in general. However, since the objective function is a homogeneous function of $x$ we still can use the approach outlined in Section 2.

Let $V(\alpha)$ denote the optimal value of problem $P(\alpha)$. Consider an optimal solution $x^{*}(\alpha)$ of $P(\alpha)$, assuming that it exists, for a fixed value of $\alpha$. Now consider $P(\hat{\alpha})$ with constraint right-hand-side $\hat{\alpha}=\kappa \alpha$ for any $\kappa>0$. Since all feasible solutions for $P(\alpha)$ can be scaled to obtain feasible solutions for $P(\hat{\alpha})$ and since these (positively) scaled solutions will have identical objective values as the corresponding solutions to $P(\alpha)$, we immediately conclude that $\kappa x^{*}(\alpha)$ is an optimal solution for $P(\hat{\alpha})$. Furthermore, optimal values $V(\alpha)$ and $V(\hat{\alpha})$ of these two problems coincide and therefore $V(\alpha)$ depends only on the sign of $\alpha$, not its magnitude. These statements continue to hold even when $V(\alpha)$ is not achieved.

Let us first consider the case when $\alpha<0$. From the argument in the previous paragraph we conclude that if we are given an $\alpha<0$, problem (20) is equivalent to (7). Therefore, using the results of Section 2, 
we conclude that for $\alpha<0$,

$$
\begin{aligned}
& V(\alpha)= \\
& \begin{cases}\sqrt{\left(\bar{v}^{2}\right)^{\mathrm{T}} Q_{2}^{-1} \bar{v}^{2}} & \text { if } e^{\mathrm{T}} Q_{2}^{-1} \bar{v}^{2}<0, \\
\sqrt{\left(\bar{v}^{2}\right)^{\mathrm{T}} Q_{2}^{-1} \bar{v}^{2}-\frac{\left(e^{\mathrm{T}} Q_{2}^{-1} \bar{v}^{2}\right)^{2}}{e^{\mathrm{T}} Q_{2}^{-1} e}} & \text { if } e^{\mathrm{T}} Q_{2}^{-1} \bar{v}^{2} \geqslant 0 .\end{cases}
\end{aligned}
$$

If $\alpha<0$ and $e^{\mathrm{T}} Q_{2}^{-1} \bar{v}^{2}<0$, the optimal solution to (20) is

$x^{*}=\frac{\alpha}{e^{\mathrm{T}} Q_{2}^{-1} \bar{v}^{2}} Q_{2}^{-1} \bar{v}^{2}$.

If $\alpha<0$ but $e^{\mathrm{T}} Q_{2}^{-1} \bar{v}^{2} \geqslant 0$, the optimal value is not achieved but we can get arbitrarily close to this value by considering solutions of the form $\hat{x}+\beta x^{1}$ where $e^{\mathrm{T}} \hat{x}=\alpha, x^{1}$ is as in (11)-(14) and with $\beta$ tending to $+\infty$. To see this, one has to evaluate the limit $\lim _{\beta \rightarrow \infty} h(\beta)$ where
So, when $\alpha>0$ the situation is reversed. If $e^{\mathrm{T}} Q_{2}^{-1} \bar{v}^{2}>0$, a positive multiple of $Q_{2}^{-1} \bar{v}^{2}$ - the optimal solution to the unconstrained version of (20)-is feasible for (20), and therefore is optimal. If $e^{\mathrm{T}} Q_{2}^{-1} \bar{v}^{2} \leqslant 0$, then the optimal value is approached by solutions of the form $\hat{x}+\beta x^{1}$ where $e^{\mathrm{T}} \hat{x}=\alpha, x^{1}$ is as in (11)-(14) and with $\beta$ tending to $+\infty$.

Finally, we note that when $\alpha=0$, the optimal solution is given by Eqs. (11)-(14) and the optimal value is

$$
\sqrt{\left(\bar{v}^{2}\right)^{\mathrm{T}} Q_{2}^{-1} \bar{v}^{2}-\frac{\left(e^{\mathrm{T}} Q_{2}^{-1} \bar{v}^{2}\right)^{2}}{e^{\mathrm{T}} Q_{2}^{-1} e}}
$$

regardless of the sign of $e^{\mathrm{T}} Q_{2}^{-1} \bar{v}^{2}$.

To summarize, we have that the optimal value of (20) is either $\sqrt{\left(\bar{v}^{2}\right)^{\mathrm{T}} Q_{2}^{-1} \bar{v}^{2}}$ (when the sign of $\alpha$ and $e^{\mathrm{T}} Q_{2}^{-1} \bar{v}^{2}$ coincide) or $h(\beta)=\frac{\left(\bar{v}^{2}\right)^{\mathrm{T}}\left(\hat{x}+\beta\left(\gamma Q_{2}^{-1} \bar{v}^{2}-\lambda Q_{2}^{-1} e\right)\right)}{\sqrt{\left(\hat{x}+\beta\left(\gamma Q_{2}^{-1} \bar{v}^{2}-\lambda Q_{2}^{-1} e\right)\right)^{\mathrm{T}} Q_{2}\left(\hat{x}+\beta\left(\gamma Q_{2}^{-1} \bar{v}^{2}-\lambda Q_{2}^{-1} e\right)\right)}}$.

After substituting the expressions for $\gamma$ and $\lambda$ and some algebraic manipulation the above limit simplifies to the following:

$\sqrt{\left(\bar{v}^{2}\right)^{\mathrm{T}} Q_{2}^{-1} \bar{v}^{2}-\left(e^{\mathrm{T}} Q_{2}^{-1} \bar{v}^{2}\right)^{2} / e^{\mathrm{T}} Q_{2}^{-1} e}$ (otherwise). As mentioned above, other than determining which

$\lim _{\beta \rightarrow \infty} \frac{\left(\bar{v}^{2}\right)^{\mathrm{T}} \hat{x}+\beta}{\sqrt{\frac{\hat{x}^{\mathrm{T}} Q_{2} \hat{x}\left(e^{\mathrm{T}} Q_{2}^{-1} e\left(\bar{v}^{2}\right)^{\mathrm{T}} Q_{2}^{-1} \bar{v}^{2}-\left(\left(\bar{v}^{2}\right)^{\mathrm{T}} Q_{2}^{-1} e\right)^{2}\right)+\beta\left(e^{\mathrm{T}} Q_{2}^{-1} e\left(\bar{v}^{2}\right)^{\mathrm{T}} \hat{x}-2\left(\bar{v}^{2}\right)^{\mathrm{T}} Q_{2}^{-1} e \alpha\right)+\beta^{2} e^{\mathrm{T}} Q_{2}^{-1} e}{e^{\mathrm{T}} Q_{2}^{-1} e\left(\bar{v}^{2}\right)^{\mathrm{T}} Q_{2}^{-1} \bar{v}^{2}-\left(\left(\bar{v}^{2}\right)^{\mathrm{T}} Q_{2}^{-1} e\right)^{2}}}}$

from which the desired conclusion easily follows.

Next, we consider the case when $\alpha>0$. In this case, problem (20) is equivalent to the following problem obtained by flipping the direction of the constraint in (7):

$$
\begin{aligned}
& \sup _{x} \frac{\bar{v}^{\mathrm{T}} x}{\sqrt{x^{\mathrm{T}} Q x}}, \\
& e^{\mathrm{T}} x>0 .
\end{aligned}
$$

Using analogous arguments to those in Section 2, we easily conclude that the optimal value of this problem as well as of (20) is given as follows:

$$
\begin{aligned}
& V(\alpha)= \\
& \begin{cases}\sqrt{\left(\bar{v}^{2}\right)^{\mathrm{T}} Q_{2}^{-1} \bar{v}^{2}} & \text { if } e^{\mathrm{T}} Q_{2}^{-1} \bar{v}^{2}>0, \\
\sqrt{\left(\bar{v}^{2}\right)^{\mathrm{T}} Q_{2}^{-1} \bar{v}^{2}-\frac{\left(e^{\mathrm{T}} Q_{2}^{-1} \bar{v}^{2}\right)^{2}}{e^{\mathrm{T}} Q_{2}^{-1} e}} & \text { if } e^{\mathrm{T}} Q_{2}^{-1} \bar{v}^{2} \leqslant 0 .\end{cases}
\end{aligned}
$$

"regime" we are in through its sign, the value of $\alpha$ has no bearing on this optimal value. This counter-intuitive conclusion appears to be an artifact of our assumption that $Q$ is positive definite and hence risk-free securities are not available. We remove this assumption in the next section and obtain more intuitive conclusions.

Now, let us go back to the two-period problem in (19). From the discussion above, we conclude that this problem is equivalent to the following problem:

$$
\sup _{x^{0}: e^{\mathrm{T}} x^{0}<0} \inf _{v^{1} \in \mathscr{U}} v\left(x^{0}, v^{1}\right)
$$

where

$$
\begin{aligned}
& v\left(x^{0}, v^{1}\right)= \\
& \begin{cases}\sqrt{\left(\bar{v}^{2}\right)^{\mathrm{T}} Q_{2}^{-1} \bar{v}^{2}} & \text { if } \frac{\left(v^{1}\right)^{\mathrm{T}} x^{0}}{e^{\mathrm{T}} Q_{2}^{-1} \bar{v}^{2}}>0, \\
\sqrt{\left(\bar{v}^{2}\right)^{\mathrm{T}} Q_{2}^{-1} \bar{v}^{2}-\frac{\left(e^{\mathrm{T}} Q_{2}^{-1} \bar{v}^{2}\right)^{2}}{e^{\mathrm{T}} Q_{2}^{-1} e}} & \text { otherwise. }\end{cases}
\end{aligned}
$$


Since the value function $v\left(x^{0}, v^{1}\right)$ depends on $x^{0}$ and $v^{1}$ only through the sign of the expression $\left(v^{1}\right)^{\mathrm{T}} x^{0}$, we have the following conclusions:

- If $e^{\mathrm{T}} Q_{2}^{-1} \bar{v}^{2}>0$, then the optimal value of (22) is $\sqrt{\left(\bar{v}^{2}\right)^{\mathrm{T}} Q_{2}^{-1} \bar{v}^{2}}$ if there exists an $x^{0}$ such that

$$
e^{\mathrm{T}} x^{0}<0 \text { and }\left(v^{1}\right)^{\mathrm{T}} x^{0}>0, \forall v^{1} \in \mathscr{U} .
$$

Otherwise, the choice of $x^{0}$ is immaterial and the optimal value is

$$
\sqrt{\left(\bar{v}^{2}\right)^{\mathrm{T}} Q_{2}^{-1} \bar{v}^{2}-\frac{\left(e^{\mathrm{T}} Q_{2}^{-1} \bar{v}^{2}\right)^{2}}{e^{\mathrm{T}} Q_{2}^{-1} e} .}
$$

The tractability of the feasibility system (23) depends on the uncertainty set $\mathscr{U}$ for $v^{1}$. If we have an ellipsoidal uncertainty set $\mathscr{U}=\left\{\bar{v}^{1}+L u:\|u\|_{2} \leqslant 1\right\}$, then (23) is equivalent to $e^{\mathrm{T}} x^{0}<0$ and $\left(\bar{v}^{1}\right)^{\mathrm{T}} x^{0}-$ $\sqrt{\left(x^{0}\right)^{\mathrm{T}} L L^{\mathrm{T}} x^{0}}>0$. See Eq. (4) and the paragraph following it in Section 1.1. This convex system can be easily resolved. Note that a feasible solution for (23) indicates a period 1 arbitrage opportunity and therefore is unlikely to exist.

- If $e^{\mathrm{T}} Q_{2}^{-1} \bar{v}^{2}<0$, then the optimal value of (22) is $\sqrt{\left(\bar{v}^{2}\right)^{\mathrm{T}} Q_{2}^{-1} \bar{v}^{2}}$ if there exists an $x^{0}$ such that

$$
e^{\mathrm{T}} x^{0}<0 \quad \text { and } \quad\left(v^{1}\right)^{\mathrm{T}} x^{0}<0, \quad \forall v^{1} \in \mathscr{U} .(24)
$$

If we have $\mathscr{U}=\left\{\bar{v}^{1}+L u:\|u\|_{2} \leqslant 1\right\}$ as above, then (24) is equivalent to $e^{\mathrm{T}} x^{0}<0$ and $\left(\bar{v}^{1}\right)^{\mathrm{T}} x^{0}+$ $\sqrt{\left(x^{0}\right)^{\mathrm{T}} L L^{\mathrm{T}} x^{0}}<0$. This, again, is a convex system and can be solved easily.

The second case we described above illustrates the anomaly caused by the lack of a riskless asset for investment in the second period. If $e^{\mathrm{T}} Q_{2}^{-1} \bar{v}^{2}<0$, in order to maximize the $\theta$ for period 2 , we try to choose an $x^{0}$ such that the value of this portfolio at the end of the first period is guaranteed to be negative! This counter-intuitive situation does not arise when we introduce riskless assets.

\section{With a riskless asset}

We use the earlier notation and now let $x_{f}^{0}$ and $x_{f}^{1}$ denote our holdings in the risk-free asset at periods 0 and 1 , and let $v_{f}^{1} \geqslant 1$ and $v_{f}^{2} \geqslant 1$ be the (deterministic) time 1 and time 2 values of a $\$ 1$ invested in the riskless asset at times 0 and 1 , respectively. The analog of problem (19) in this setting is

$\sup _{x^{0}, x_{f}^{0}: e^{\mathrm{T}} x^{0}+x_{f}^{0}<0} \inf _{v^{1} \in \mathscr{U}} \sup _{\theta, x^{1}, x_{f}^{1}} \theta$

s.t.

$$
\begin{aligned}
& e^{\mathrm{T}} x^{1}+x_{f}^{1}=\left(v^{1}\right)^{\mathrm{T}} x^{0}+v_{f}^{1} x_{f}^{0}, \\
& \left(\bar{v}^{2}\right)^{\mathrm{T}} x^{1}+v_{f}^{2} x_{f}^{1}-\theta \sqrt{\left(x^{1}\right)^{\mathrm{T}} Q_{2} x^{1}} \geqslant 0 .
\end{aligned}
$$

As before, we focus on the inner maximization problem: Given $\alpha=\left(v^{1}\right)^{\mathrm{T}} x^{0}+v_{f}^{1} x_{f}^{0}$, we solve:

$$
\begin{aligned}
& \sup _{\theta, x^{1}, x_{f}^{1}} \theta \text { s.t. } e^{\mathrm{T}} x^{1}+x_{f}^{1}=\alpha, \\
& \left(\bar{v}^{2}\right)^{\mathrm{T}} x^{1}+v_{f}^{2} x_{f}^{1}-\theta \sqrt{\left(x^{1}\right)^{\mathrm{T}} Q_{2} x^{1}} \geqslant 0 .
\end{aligned}
$$

If $\alpha>0$, i.e., if we have a positive-valued portfolio at the end of period 1 , then, the inner maximization problem is unbounded as we can choose $x^{1}=0, x_{f}^{1}=\alpha$ and all $\theta$ 's will be feasible for the problem. In other words, if our position (which had a negative value initially) reaches a positive value, we can quit gambling and put all our money in the riskless asset to guarantee that we make money at the end.

Now consider the case when $\alpha<0$. In this case, there is no feasible solution to (26) with $x^{1}=0$ and $\theta>0$, therefore, we do not need to worry about division by zero and rewrite (26) as

$$
\begin{aligned}
& \sup _{x^{1}, x_{f}^{1}} \frac{\left(\bar{v}^{2}\right)^{\mathrm{T}} x^{1}+v_{f}^{2} x_{f}^{1}}{\sqrt{\left(x^{1}\right)^{\mathrm{T}} Q_{2} x^{1}}}, \\
& e^{\mathrm{T}} x^{1}+x_{f}^{1}=\alpha .
\end{aligned}
$$

Using the constraint we eliminate $x_{f}^{1}$ and obtain the following unconstrained problem:

$\sup _{x^{1}} f\left(x^{1}\right):=\frac{\left(\bar{v}^{2}-v_{f}^{2} e\right)^{\mathrm{T}} x^{1}+\alpha v_{f}^{2}}{\sqrt{\left(x^{1}\right)^{\mathrm{T}} Q_{2} x^{1}}}$.

Observe that for any $x^{1}$ and for any $\beta>1$,

$f\left(\beta x^{1}\right)=f\left(x^{1}\right)-\left(1-\frac{1}{\beta}\right) \frac{\alpha v_{f}^{2}}{\sqrt{\left(x^{1}\right)^{\mathrm{T}} Q_{2} x^{1}}}>f\left(x^{1}\right)$. 
So, for any solution $x^{1}$, we can always improve the solution by scaling it up, and therefore, the supremum in (27) is never achieved. Note that

$\lim _{\beta \rightarrow \infty} f\left(\beta x^{1}\right)=\frac{\left(\bar{v}^{2}-v_{f}^{2} e\right)^{\mathrm{T}} x^{1}}{\sqrt{\left(x^{1}\right)^{\mathrm{T}} Q_{2} x^{1}}}$.

Thus, the supremum value of (27) is the same as the supremum value of the following problem with the homogeneous objective function: $\sup _{x^{1}}\left[\left(\bar{v}^{2}-v_{f}^{2} e\right)^{\mathrm{T}} x^{1}\right] /\left[\sqrt{\left(x^{1}\right)^{\mathrm{T}} Q_{2} x^{1}}\right]$. We can solve this problem by introducing a normalizing constraint as we did before and obtain that the optimal solution ray is: $x^{1}=\beta Q_{2}^{-1}\left(\bar{v}^{2}-v_{f}^{2} e\right), \beta>0$. Note that these are maximum-Sharpe ratio portfolios. The optimal objective value along this ray is $\sqrt{\hat{r}^{\mathrm{T}} Q_{2}^{-1} \hat{r}}$ with $\hat{r}=\bar{v}^{2}-v_{f}^{2} e$.

Combining our conclusions, we have that (25) is equivalent to

$$
\sup _{x^{0}, x_{f}^{0}: e^{\mathrm{T}} x^{0}+x_{f}^{0}<0} \inf _{v^{1} \in \mathscr{U}} v\left(x^{0}, x_{f}^{0}, v^{1}\right),
$$

where

$$
\begin{aligned}
& v\left(x^{0}, x_{f}^{0}, v^{1}\right) \\
& \quad= \begin{cases}+\infty & \text { if }\left(v^{1}\right)^{\mathrm{T}} x^{0}+v_{f}^{1} x_{f}^{0}>0, \\
\sqrt{\hat{r}^{\mathrm{T}} Q_{2}^{-1} \hat{r}} & \text { otherwise. }\end{cases}
\end{aligned}
$$

From this, we immediately obtain the optimal solution for the two-period problem: If there is a period 1 arbitrage opportunity, i.e., if there exists $x^{0}, x_{f}^{0}$ such that

$$
\begin{aligned}
& e^{\mathrm{T}} x^{0}+x_{f}^{0}<0 \text { and }\left(v^{1}\right)^{\mathrm{T}} x^{0}+v_{f}^{1} x_{f}^{0}>0, \\
& \forall v^{1} \in \mathscr{U},
\end{aligned}
$$

then take this position at time 0 and move everything to the risk-free asset at time 1 . If not, then $x^{0}, x_{f}^{0}$ do not matter for the two-period maximum$\theta$ problem (but, of course, one may choose these variables in order to maximize the probability that $\left(v^{1}\right)^{\mathrm{T}} x^{0}+v_{f}^{1} x_{f}^{0}>0$, provided that we have a probability distribution for $v^{1}$ ). Once we reach time 1 , if we observe that $\left(v^{1}\right)^{\mathrm{T}} x^{0}+v_{f}^{1} x_{f}^{0}>0$, we again move everything to the risk-free asset. Otherwise, we can take a position that comes arbitrarily close to the maximum$\theta$ value of $\sqrt{\hat{r}^{\mathrm{T}} Q_{2}^{-1} \hat{r}}$.

\section{References}

[1] A. Ben-Tal, A. Goryashko, E. Guslitzer, A. Nemirovski, Adjustable robust solutions of uncertain linear programs, Math. Program. 99 (2) (2004) 351-376.

[2] A. Ben-Tal, A. Nemirovski, Robust convex optimization, Math. Oper. Res. 23 (1998) 769-805.

[3] A. Ben-Tal, A. Nemirovski, Robust solutions to uncertain linear programming problems, Oper. Res. Lett. 25 (1999) 113.

[4] A. Ben-Tal, A. Nemirovski, Robust solutions of linear programming problems contaminated with uncertain data, Math. Program. 88 (2000) 411-424.

[5] D. Duffie, Dynamic Asset Pricing Theory, Princeton University Press, Princeton, NJ, 1992.

[6] D. Duffie, J. Pan, An overview of value at risk, J. Derivatives 4 (1997) 7-49.

[7] L. El Ghaoui, M. Oks, F. Oustry, Worst-case value-at-risk and robust portfolio optimization: a conic programming approach, Oper. Res. 51 (2003) 543-556.

[8] D. Goldfarb, G. Iyengar, Robust portfolio selection problems, Math. Oper. Res. 28 (1) (2003) 1-38.

[9] E. Guslitzer, Uncertainty-immunized solutions in linear programming, Masters Thesis. Technion. Haifa, Israel, 2002.

[10] J. Ingersoll, Theory of Financial Decision Making, Rowman \& Littlefield, Savage, Maryland, 1987.

[11] M.S. Lobo, L. Vandenberghe, S. Boyd, H. Lebret, Applications of second-order cone programming, Linear Algebra Appl. 284 (1998) 193-228.

[12] M.Ç. Pınar, Minimum risk arbitrage with risky financial contracts, Technical Report, Department of Industrial Engineering, Bilkent University, Ankara, Turkey, 2003.

[13] S.R. Pliska, Introduction to Mathematical Finance, Blackwell Publishers, Oxford, 1997.

[14] P. Whittle, Optimization under Constraints. Theory and Applications of Nonlinear Programming, Wiley-Interscience, New York, 1971. 